Résumés des conférences et travaux

\title{
Histoire monétaire du monde romain
}

\section{Antony Hostein}

\section{OpenEdition \\ Journals}

Édition électronique

URL : https://journals.openedition.org/ashp/2348

DOI : $10.4000 /$ ashp.2348

ISSN : 1969-6310

Éditeur

Publications de l'École Pratique des Hautes Études

\section{Édition imprimée}

Date de publication : 1 septembre 2018

Pagination : 133-140

ISSN : 0766-0677

\section{Référence électronique}

Antony Hostein, "Histoire monétaire du monde romain », Annuaire de l'École pratique des hautes études (EPHE), Section des sciences historiques et philologiques [En ligne], 149 | 2018, mis en ligne le 11 juillet 2018, consulté le 03 août 2021. URL : http://journals.openedition.org/ashp/2348 ; DOI : https:// doi.org/10.4000/ashp.2348 


\title{
HISTOIRE MONÉTAIRE DU MONDE ROMAIN
}

\author{
Directeur d'études : M. Antony Hosteın
}

Programme de l'année 2016-2017 : I. Conférence inaugurale. - II. Les monnaies d'Alexandrie de Troade, d'Antonin le Pieux à Gallien. - III. Recherches sur les médaillons d'époque antonine.

\section{Conférence inaugurale}

L'intitulé de la direction d'études ainsi que l'orientation donnée aux premières conférences ont été expliqués au cours d'une séance introductive.

Dans l'une des dernières contributions parues de son vivant, un recueil d'hommages en l'honneur du docteur Bastien (1912-2010), formulant plusieurs réflexions sur le travail du corpus pour les historiens, Jean-Pierre Callu (1929-2014) a rappelé à son lecteur les deux principaux avantages de la numismatique, en une formule lapidaire dont lui seul avait le secret : «Le numérique et le continu, ces deux atouts propres à la numismatique ». Dans cette phrase, un mot s'entendait dans un double sens qui a peut-être échappé à son auteur, celui de «numérique » qui, en plus de signifier «nombre », peut aussi se rapporter aux nouvelles technologies liées à internet, comme dans l'expression à l'ère du «numérique ». Ce grand savant et numismate n'ignorait pas pour autant l'importance des outils informatiques puisque dans le même article il concluait sa réflexion par ces mots :

En quelques années de progrès cumulatifs, l'outil informatique promet d'avaler les délais. Pourquoi la numismatique n'en profiterait-elle pas ? ${ }^{1}$.

La conférence « Histoire monétaire du monde romain », si elle garde un intitulé classique, mais dont le mot numismatique est absent, est inaugurée dans un contexte où les travaux sur la monnaie antique connaissent de profonds bouleversements grâce aux nouveaux outils informatiques mis à disposition des chercheurs.

Sur le travail de catalogage stricto sensu, la révolution, le terme n'est pas trop fort, vient du temps gagné d'abord, en amont mais aussi en aval : plus de frottis peu lisibles, plus de fragiles moulages photographiés sur pellicule argentique et organisés sur des planches-contacts : la pièce peut être photographiée, détourée, traitée, mise à l'échelle par un logiciel de traitement de l'image, prête à l'étude et à l'impression. De surcroît, l'image numérique, grâce au zoom, peut être explorée et examinée pour faire apparaître des détails illisibles ou peu reconnaissables. La mise en ligne du corpus permet ensuite de rendre accessible à plus vaste échelle une documentation sérielle, depuis n'importe quel poste connecté à internet. Le corpus bénéficie ainsi d'une exposition large, mise à profit par exemple dans les retours et remarques des internautes. Cette mise en ligne, associée à la publication papier qu'elle n'abolit pas, permet par ailleurs de traiter des questions numismatiques et historiques entravées d'ordinaire par l'arbitraire des découpages que subit la masse documentaire répartie selon une

1. J.-P. Callu, « Les corpus numismatiques et l'historien », $R N, 2014$, p. 21-29, p. 28 pour la citation. 
logique chronologique, par volume. Le site internet rétablit ainsi la continuité historique rompue par la césure du volume. Enfin, la mise en ligne de la documentation, partielle ou complète, autorise des requêtes transversales sur des thèmes variés. L'outil informatique offre aussi la possibilité de distribuer instantanément et spatialement, sur un plan ou une carte, les requêtes effectuées. La carte qui en résulte ne doit pas être considérée comme une simple illustration du phénomène examiné, mais bien comme une façon de penser autrement ce qui s'y trouve fixé.

L'intégration massive des données dans une base ouvre des perspectives infinies dans les domaines voisins, par le jeu du croisement des informations. Beaucoup de travail reste à faire, mais les efforts consentis pour garantir la pérennité des adresses des objets mis en ligne (URI stables), l'uniformité des données (grâce à l'ontologie transnationale Nomisma ${ }^{2}$ ) ainsi que l'interopérabilité des bases (Données ouvertes et liées - DOL), aussi bien en histoire, en numismatique qu'en archéologie vont permettre d'aborder des questions anciennes sous des angles originaux. Ce qui d'ordinaire mettait des heures à être réalisé à la main, en bibliothèque ou dans un musée - catalogues, cartes, tableaux, statistiques -, pourra être effectué instantanément, libérant autant de temps pour la réflexion, les lectures et l'interprétation ${ }^{3}$. À plus d'un titre, les changements en cours dans la numismatique demeurent comparables à ceux intervenus dans la seconde moitié du $\mathrm{XX}^{\mathrm{e}}$ siècle grâce à l'apport des statistiques et des nouvelles méthodes d'analyses métalliques.

Les recherches et enseignements conduits dans la conférence ont vocation à faire converger deux grandes traditions de la numismatique développées aux Hautes Études depuis les années 1930, dans une approche globale de la monnaie (histoire économique, politique, sociale et culturelle) visant à construire le «fait monétaire ». La première tradition est illustrée par Pierre le Gentilhomme (1909-1947), Jean Lafaurie (1914-2008) ou Jean-Pierre Callu; elle se fonde sur le catalogue exhaustif et le traitement sériel et continu d'ensembles monétaires afin d'en dégager de grandes tendances historiques ${ }^{4}$; la seconde, héritée des travaux de Louis Robert (1904-1985), repose sur l'examen minutieux des légendes et des images des monnaies civiques à légende grecque (ou latine), analyse qui ouvre la voie à de nombreuses recherches sur la vie des communautés de l'Orient romain. À ces deux influences s'ajoutent celle exercée par Michel Amandry qui a fondé avec Andrew Burnett l'entreprise du Roman Provincial Coinage, et celle de l'école anglaise de l'archéologie numismatique (ou

2. Placé sous le patronage de l'International Numismatic Council (INC), le projet Nomisma a pour vocation principale de fournir des représentations digitales stables pour les données numismatiques en suivant les principes du web sémantique (linked web), en offrant des données en licence ouverte (open data) et en mettant en relation différentes sources entre elles. Conçu au départ par des chercheurs de l'American Numismatic Society et de 1'Institute for Advanced Studies of the Ancient World de 1'université de New York, ce projet international associe de très nombreuses institutions académiques ou muséales : voir http://nomisma.org.

3. L'apport de ces nouvelles approches numériques est fondamental. On peut en prendre la mesure à la lecture de 1'article de D. Wigg-Wolf, F. Duyrat, « La révolution des Linked Open Data en numismatique : les exemples de nomisma.org et Online Greek Coinage », ISTE OpenScience, 2017-1, en ligne à l'adresse suivante : https://www.openscience.fr/La-revolution-des-Linked-Open-Data-en-numismatique-Les-exemples-de-nomisma-org

4. Voir l'article cité supra de J.-P. Callu, « Les corpus numismatiques et l'historien », RN, 2014, p. 21-29. 
de la numismatique archéologique, c'est selon), dont l'une des figures majeures est Richard Reece. Depuis les années 1970, cette école a beaucoup apporté à la discipline en étudiant la monnaie dans sa dimension concrète d'objet archéologique, en prenant en compte avec précision le contexte stratigraphique de chaque spécimen découvert lors de fouilles, sans le réduire à un simple marqueur de datation ${ }^{5}$. Le lancement en 2011 d'une revue au titre programmatique, le Journal of Archaeological Numismatic (abrégé $J A N$ ), est venu consacrer ce rameau récent de la numismatique.

C'est dans ce cadre méthodologique renouvelé que l'étude de deux facettes en particulier de l'histoire monétaire et de la numismatique est envisagée, en s'efforçant toujours « de tirer la numismatique de son isolement et de la faire servir à l'étude de la civilisation antique $»^{6}$. L'approche adoptée s'inscrit dans les travaux de recherche passés du Directeur d'études. Deux orientations principales seront suivies : la première, dans le prolongement des travaux inaugurés durant la thèse ${ }^{7}$, concerne la vie des communautés civiques de l'Empire romain, à travers divers éclairages comme l'étude des institutions, de la culture matérielle, des pratiques religieuses, ou encore des identités locales; la seconde porte sur les relations qu'entretenaient ces mêmes communautés locales avec le pouvoir impérial, en s'intéressant aux agents et aux pratiques institutionnelles. Une attention privilégiée sera portée aux époques de transition, que ce soit le passage entre la République et l'Empire, ou entre le Hautet le Bas-Empire. L'accent sera placé sur des cas d'études choisis dans différentes aires géographiques de l'Empire romain (Gaules, Germanies, Afrique, provinces de l'Orient hellénophone).

Quant à la documentation destinée à fonder ces réflexions, elle sera puisée à deux sources principales : d'abord des séries réunies pour les volumes en cours de réalisation du Roman Provincial Coinage, en particulier ceux sur les Sévères et sur le milieu du III $^{\mathrm{e}}$ siècle; ensuite des dépôts et monnaies de sites inédits ou publiés chaque année, en Gaule principalement mais aussi dans les autres territoires qui formaient l'ancien Empire romain. Fidèle à la tradition et aux principes énoncés par les fondateurs de l'École pratique, l'enseignement et les recherches conçues dans le cadre de la conférence se feront avec les objets monétaires, examinés directement, dans des collections publiques et privées, dans des musées ou des réserves de centres archéologiques.

Après avoir tiré profit des textes littéraires, des inscriptions ou encore des sources matérielles, le temps est donc venu de prolonger les travaux sur les cités et le pouvoir impérial en les reliant davantage à l'histoire monétaire. Si des pistes ont été explorées avec des collègues archéologues sur la crise et les transformations des cités

5. Voir par exemple l'ouvrage collectif fondateur de cette école : J. Casey, R. Reece (éd.), Coins and the Archaeologist, Oxford, 1974.

6. C'est en ces termes que l'historien et épigraphiste Louis Robert dressait le bilan de plusieurs cours dispensés dans sa conférence aux Hautes Études en 1945-1946 : Opera Minora Selecta. IV, Amsterdam, 1974, p. 91. Dans cette même conférence, traitant d'austères questions d'onomastique, il aimait à rappeler à son auditoire qu'il ne cherchait pas seulement à faire une histoire des noms, mais à écrire l'histoire par les noms. Cette réflexion vaut également pour la monnaie.

7. A. Hostein, La cité et l'empereur. Les Éduens dans l'Empire romain d'après les Panégyriques latins, Paris, 2012. 
gallo-romaines au $\mathrm{III}^{\mathrm{e}}$ siècle ${ }^{8}$, une documentation peu étudiée sous cet angle a retenu l'attention durant la thèse : il s'agit des monnayages provinciaux ou civiques, longtemps appelés " impériales grecques », déjà évoqués à plusieurs reprises à propos du projet du Roman Provincial Coinage. Pour mémoire, cette documentation regroupe les monnaies frappées à l'initiative de communautés locales, par opposition au monnayage impérial produit dans des ateliers officiels placés sous le contrôle du pouvoir central. Ces émissions s'interrompent en Occident sous l'empereur Claude (41-54 apr. J.-C.) mais se prolongent en Orient jusque dans les années 270 apr. J.-C. Espèces composées principalement de pièces de bronze et plus rarement d'argent, destinées à circuler à une échelle régionale, elles ont souvent été émises en quantité limitée, le pouvoir romain conservant le monopole de l'or et la frappe massive du métal argenté pour le financement des guerres et des grands travaux. Dans le cadre du plan de travail défini supra, le principal intérêt des monnayages provinciaux, en particulier ceux du III $^{\mathrm{e}}$ siècle, réside dans la possibilité d'articuler l'étude des cités de l'Orient avec celle du pouvoir impérial, sans jamais perdre de vue aucune des branches qui composent la numismatique : étude des monnaies de site ou de fouilles; des dépôts monétaires; histoire économique ou politique; histoire de l'art et des représentations. La monnaie est d'autant plus importante que, à compter des années 240, les témoignages littéraires et épigraphiques tendent à se raréfier.

Au sujet du Roman Provincial Coinage, l'une des dernières grandes entreprises éditoriales pour l'histoire romaine impériale, dans la tradition du Corpus Inscriptionum Latinarum ou encore du Roman Imperial Coinage, on précisera qu'après le volume IX consacré aux années 249-253, paru en 2016 et mis en ligne depuis $2015^{9}$, le prochain catalogue dont la coordination va être assurée par le Directeur d'étude et Jérôme Mairat, conservateur à l'Ashmolean Museum d'Oxford, est le volume V-2. Ce volume comprend les émissions des cités de la province d'Asie sous les premiers Sévères, de 193 à 222 apr. J.-C. S'agissant d'un des plus riches en nombre d'ateliers monétaires et de spécimens, sa réalisation va impliquer un travail opiniâtre de collecte et d'identification des pièces qu'il est prévu d'entamer en 2018 avec pour objectif la mise en ligne de la documentation à l'horizon 2022. En attendant d'entreprendre ce travail, le riche matériau accumulé pour le volume IX du $R P C$ va être employé à différentes enquêtes sur le règne de l'empereur Trajan Dèce (249-251) et sur les identités civiques locales.

Le second axe de recherche porte sur l'étude des trésors et des dépôts monétaires antiques mis au jour en France. Ce projet collectif, qui s'appuie sur un financement du CNRS ${ }^{10}$, est destiné pour partie à prolonger l'entreprise inachevée des Trésors

8. Par exemple dans A. Hostein, M. Kasprzyk, «Une communauté civique face à la crise : la civitas Aeduorum dans la seconde moitié du $\mathrm{III}^{\mathrm{e}}$ siècle apr. J.-C. (approches archéologique et historique) », dans C. Berrendonner, M. Cébeillac-Gervasoni, L. Lamoine (éd.), Le quotidien municipal, II. Gérer les territoires, les patrimoines et les crises, Clermont-Ferrand, 2012, p. 519-542, ou encore dans A. Hostein, M. Joly, M. Kasprzyk, P. Nouvel, «Sanctuaires et pratiques religieuses du III s. au v s. apr. J.-C. dans le Centre-Est de la Gaule (Lugdunensis I et Maxima Sequanorum) ", Gallia, 71-1, 2014, p. 187-218.

9. Pour une présentation du projet, voir http://rpc.ashmus.ox.ac.uk/

10. Projet CNRS-PICS n 7338, « Trésors Monétaires de la Gaule - TréMoG (2016-2018) ». 
antiques de la France (abrégés $T A F)^{11}$. Un inventaire aussi exhaustif que possible des dépôts découverts dans plusieurs secteurs mal documentés de la Gaule romaine mais riches en données anciennes et récentes va être dressé. Les régions concernées dans un premier temps sont le Centre, la Bourgogne et la Franche-Comté qui correspondaient approximativement dans l'Antiquité aux territoires des Bituriges, des Éduens, des Lingons et des Séquanes. Les dépôts recensés, composés pour l'écrasante majorité d'entre eux de monnaies impériales, seront intégrés individuellement par le biais d'une application en ligne (web application) dans une base de données conçue dans le cadre du projet Coin Hoards of the Roman Empire (Oxford, dir. C. Howgego, A. Wilson) ${ }^{12}$. Mené en collaboration avec des archéologues de l'INRAP et des universités de Bourgogne et de Franche-Comté, il va donner l'occasion de croiser la documentation numismatique rassemblée avec l'ensemble des données archéologiques enregistrées pour ces régions dans des systèmes d'information géographique (SIG), en particulier les monnaies de site, les trouvailles isolées de pièces d'or et la documentation céramique. L'enquête vise à caractériser à une échelle fine, dans une perspective diachronique et de la façon la plus complète qui soit, le degré de monétarisation des territoires étudiés et à identifier très précisément les types de monnaies en circulation en fonction des statuts des sites où elles ont été découvertes. Ainsi, dans ces régions de la Gaule, à travers la monétarisation de l'économie et les faciès des monnaies en circulation, envisagés sur la longue durée et selon différents critères d'analyse (sites ruraux ou urbains, régions centrales ou périphériques, présence d'activités agricoles ou artisanales, etc.), des sujets d'histoire générale seront traités, en particulier la question de l'intégration des sociétés provinciales dans la romanité, processus qualifié de « romanisation » par les historiens de l'Antiquité.

En 2016-2017, la conférence a porté sur deux thèmes principaux : d'une part l'apport des monnaies provinciales à notre connaissance de l'histoire politique, institutionnelle et culturelle de la « crise du $\mathrm{II}^{\mathrm{e}}$ siècle », et d'autre part les usages de la monnaie dans les villes et les campagnes des Trois Gaules entre le $\mathrm{I}^{\mathrm{er}} \mathrm{s}$. av. et le $\mathrm{V}^{\mathrm{e}} \mathrm{s}$. apr. J.-C. À côté d'une approche numismatique classique fondée sur le classement et la typologie, l'accent a été mis sur les pièces découvertes en contexte archéologique. La monnaie a été confrontée aux autres sources disponibles (textes littéraires, inscriptions, papyrus) et en tenant compte des données récentes publiées par les archéologues. Plusieurs séances ont été consacrées à la lecture critique des publications suivantes : U. Babusiaux, A. Kolb (éd.), Das Recht der Soldatenkaiser, Berlin, Munich, Boston, 2015; R. Bennett, Local Elites and Local Coinage, Londres, 2014; A. Filges, Münzbild und Gemeinschaft. Die Prägungen der römischen Kolonien in Kleinasien, Bonn, 2015.

11. Entre 1982 et 1993 , ce projet coordonné par les regrettés Daniel Nony et Xavier Loriot a permis de publier neuf volumes, couvrant les régions suivantes : I. Poitou-Charentes et Limousin; II. Nord-Pasde-Calais, III. Pays de la Loire, IV. Haute-Normandie, V. Rhône-Alpes, VI. Aquitaine, VII. Auvergne, VIII. Picardie et IX. Île-de-France.

12. Sur le projet, voir http://oxrep.classics.ox.ac.uk/coin_hoards_of_the_roman_empire_project 


\section{Les monnaies d'Alexandrie de Troade, d'Antonin le Pieux à Gallien}

Le corpus numismatique étudié a été celui d'Alexandrie de Troade. L'histoire de la ville a d'abord été présentée, à travers les noms successifs qui lui ont été donnés (Antigoneia, Alexandreia-Alexandrie, colonia Augusta Troadensis ou Troas) depuis sa fondation par synoecisme sous Antigone le Borgne jusqu'à la visite de l'empereur Caracalla, en passant par la déduction coloniale sous Auguste. Une attention particulière a été portée à la présentation de la Troade, dans des perspectives de géographie historique et d'historiographie.

Les monnayages de bronze émis entre les règnes d'Antonin et de Gallien ont été analysés de manière approfondie à partir du corpus de référence, désormais ancien, publié par Arthur R. Bellinger ${ }^{13}$ et des données réunies en 2016 dans le $R P C$ IX (Trajan Dèce-Uranius Antoninus, de 249 à 254 de notre ère), auxquels il convient d'ajouter des travaux récents, en particulier deux articles écrits par Tommaso Lucchelli et Zeynep Çismely Ögün ${ }^{14}$. Ont été passés en revue le volume et le rythme des émissions, les légendes et l'iconographie monétaires, et enfin des questions d'ordre métrologique.

L'examen de types rares qui renvoient au passé grec de la ville et de sa région a souligné combien l'identité locale officielle était complexe et différente de celle des autres colonies romaines de l'Orient hellénophone, confirmant et précisant là des observations formulées par Peter Weiß et Axel Filges ${ }^{15}$. L'analyse a porté principalement sur les types représentant d'une part le dieu topique Apollon Smintheus (« aux rats »), dont le sanctuaire se trouvait au Sud du territoire d'Alexandrie de Troade, et d'autre part la geste d'Alexandre le Grand représenté seul ou bien accompagné du dieu civique ou de Bucéphale.

Enfin, un débat ancien a pu être tranché au sujet de la datation des émissions des monnaies sans effigie impériale au droit, appelées communément «pseudo-autonomes », en dépit du caractère inapproprié de cette terminologie ${ }^{16}$. Un inventaire provisoire de ces monnayages abondants aussi bien dans les collections publiques que dans les ventes aux enchères des dix dernières années a permis d'accroitre considérablement la documentation réunie par Arthur R. Bellinger qui demeurait lacunaire sur ce point. En s'appuyant sur ces séries nombreuses et en s'aidant des analyses stylistiques et métrologiques, ont été mises en évidence plus de quinze liaisons de coins entre des monnaies sans effigie impériale et des pièces portant au droit ou bien les

13. A. R. Bellinger, Troy. The Coins, Princeton, 1961.

14. T. Lucchelli, "Ricerche sulla monetazione di Alexandria Troas: le "emissioni civiche" ", RIN, 108, 2007, p. 169-218 et Z. Çizmeli-Öğün, «Les trouvailles monétaires du sanctuaire d'Apollon Smintheus ", dans C. Alfaro Asins et al. (éd.), XIII Congreso internacional de numismática: Actas - Proceedings - Actes, 2 vol., Madrid, 2005, p. 545-550.

15. P. Weiß, «Alexandria Troas : Griechische Traditionen und Mythen in einer römischen Colonia », dans E. Schwertheim, H. Wiegartz (éd.), Die Troas. Neue Forschungen zu Neandria und Alexandria Troas, II. Asia Minor Studien 22, Bonn, 1996, p. 157-173 et A. Filges, Münzbild und Gemeinschaft. Die Prägungen der römischen Kolonien in Kleinasien, Bonn, 2015.

16. A. Hostein, J. Mairat, « Les monnayages "pseudo-autonomes" frappés au milieu du III siècle de notre ère », dans A. Bourgeois, C. Brenot, M. Christol, S. Demougin (éd.), Singularem modestiam. Hommages à Xavier Loriot, Bordeaux, 2015, p. 107-124. 
portraits de Trébonien Galle et de Volusien (251-253), ou bien ceux de Valérien et de Gallien (253-260).

Ces liaisons de coins, dont l'inventaire demeure encore incomplet, démontrent de manière irréfutable que les émissions de bronzes civiques d'Alexandrie de Troade ont été extrêmement abondantes dans les années 250 de notre ère. Dans le RPC IX, l'atelier figure dans la liste des dix premiers centres émetteurs par le nombre de spécimens et de coins recensés ${ }^{17}$. Comment expliquer cette situation originale, qui place Alexandrie de Troade au rang d'atelier régional sur le même pied que Viminacium ou Antioche de Pisidie à la même époque? À défaut de pouvoir apporter une réponse définitive, il convient de mettre en relation cette production massive avec le contexte géopolitique et militaire des provinces et des cités situées de part et d'autre des détroits, au cours d'une décennie profondément marquée par les guerres et les incursions des Goths ${ }^{18}$.

\section{Recherches sur les médaillons d'époque antonine}

Dans le cadre d'une enquête pluriannuelle consacrée à la monnaie en Gaule romaine entre le $\mathrm{I}^{\mathrm{er}} \mathrm{S}$. av. et le $\mathrm{V}^{\mathrm{e}} \mathrm{s}$. apr. J.-C. ${ }^{19}$, l'accent a été mis en 2016-2017 sur les « médaillons » d'époque antonine mis au jour en contexte archéologique.

La notion même de médaillon, parce qu'elle comporte de nombreuses ambiguïtés, a été discutée et critiquée. Les principaux travaux et corpora publiés ont été passés en revue, en particulier l'ouvrage fondateur de Francesco Gnecchi (1847-1919) et la monographie récente de Peter Franz Mittag ${ }^{20}$. L'attention a porté sur l'atelier de Rome où ces pièces étaient frappées, sur le volume et le rythme des émissions entre les règnes d'Hadrien et de Commode, sur les liens iconographiques et programmatiques de ces médaillons avec le numéraire commun de bronze marqué SC (sesterces, dupondii, as), et enfin sur la fonction festive et commémorative de ces espèces qui s'insèrent bel et bien dans la métrologie officielle.

Dans un second temps, ont été recensés les médaillons découverts en contexte archéologique. L'inventaire, fondé sur des recherches passées ${ }^{21}$ et des découvertes récentes a concerné en priorité les provinces des Trois Gaules et les Germanies. En dépit du naufrage de la documentation et de son inégale qualité, trois contextes principaux ont ainsi été mis en évidence : (i) en milieu urbain, dans des structures datées du $\mathrm{III}^{\mathrm{e}}$ siècle qui témoignent de la présence d'élites fortunées dans ces lieux (Autun,

17. Voir RPC IX, p. 120-122.

18. Pour une première interprétation du phénomène, voir A. Hostein, J. Mairat, « Les monnaies d'Alexandrie de Troade au milieu du III $^{\mathrm{e}}$ siècle : liaisons de coins inédites », dans R. Bland, D. Calomino (éd.), Studies in Ancient Coinage in Honour of Andrew Burnett, Londres, 2015, p. 223-235.

19. Voir nos remarques formulées supra, n. 10 et texte correspondant.

20. F. Gnecchi, I Medaglioni Romani, 3 vol., Milan, 1912 et P. F. Mittag, Römische Medaillons, Caesar bis Hadrian, Stuttgart, 2012 ( $2^{\mathrm{e}}$ éd. corr.).

21. M. Amandry, «Un médaillon de Commode découvert à Reims », BSFN, 47-6, 1992, p. 327-330; J.-M. Doyen, Économie, monnaie et société à Reims sous l'Empire romain. Recherches sur la circulation monétaire en Gaule septentrionale intérieure, Reims, 2007, p. 391-406 et M. C. Molinari, «Alcune Riflessioni sui ritrovamenti di medaglioni e di grandi bronzi provinciali a Roma », RIN, 103, 2002, p. 203-217. 
Reims, Cologne); (ii) dans des dépôts monétaires mixtes de grande taille, datés de l'époque de l'Empire gaulois (entre 260 et 274), et qui contiennent monnaies et bijoux thésaurisés («trésors » d'Éauze et de Talmont-Saint-Hilaire en Vendée); (iii) enfin dans des sépultures de la fin du $\mathrm{III}^{\mathrm{e}}$ et du $\mathrm{IV}^{\mathrm{e}}$ siècle où les médaillons côtoient d'autres monnaies et / ou des éléments de mobilier.

L'attention portée aux médaillons mis au jour en contexte funéraire a conduit à s'intéresser à la présence de ces espèces dans les catacombes de Rome à la même période (fin du $\mathrm{III}^{\mathrm{e}}$-début du $\mathrm{IV}^{\mathrm{e}}$ siècle), afin d'en tirer, par le jeu des comparaisons, des réflexions méthodologiques. Le dossier des trouvailles monétaires de la catacombe de Saint-Pamphyle, publié par Camillo Serafini en 1938, a été reconsidéré22. Dans cette catacombe située sur la via Salaria vetus (aujourd'hui via Paisiello), fouillée dans les années 1920, et qui a échappé aux dégradations et aux spoliations grâce aux alluvionnements qui en ont scellé les galeries, il est permis, à condition de rétablir l'objet monétaire dans son micro-contexte (étage, galerie, sépulture - loculus, cubiculum, arcosolium), de proposer différentes interprétations de l'usage qui était fait en ces lieux des monnaies. Fixées dans le mortier encore frais des sépultures, placées bien en vue des visiteurs, elles pouvaient être de simples éléments de décoration au message plus ou moins élaboré, des moyens de signaler et de singulariser la tombe, et peutêtre aussi des vestiges d'offrandes ou de paiements (réels ou symboliques). L'enquête n'ayant reposé que sur l'exploitation d'archives et de publications anciennes, il conviendra de la prolonger par un séjour romain et une visite aux musées du Vatican, afin de consulter directement le matériel recueilli et de visiter les galeries des catacombes où les empreintes de monnaies demeurent encore visibles.

22. C. Serafini, « Saggio intorno alle monete e medaglioni antichi ritrovati nelle catacombe di Pamfilo sulla Via Salaria Vetus in Roma », dans Scritti in onore di Bartolomeo Nogara raccolti in occasione del suo lxx anno, Cité du Vatican, 1937, p. 421-443. 